\title{
Hipoglucemia hiperinsulinémica en el adulto. Reporte de un caso de nesidioblastosis y revisión de la literatura
}

\author{
Hyperinsulinemic hypoglycemia in adults. Nesidioblastosis case report and review of the \\ literature
}

\section{Saúl E. Torres-Arano*, Noelia Fernández-Baez², Manuel G. Torres-González, Itzel G. González-Cabrera ${ }^{3}$ y Federico López-Rosales ${ }^{1}$}

${ }^{1}$ Servicio de Cirugía General, Hospital Regional de Alta Especialidad de la Península de Yucatán, Mérida, Yucatán; '2Servicio de Coloproctología, Hospital General de México, Ciudad de México; ${ }^{3}$ Departamento de Investigación, Hospital Regional de Alta Especialidad de la Península de Yucatán, Mérida, Yucatán. México

\section{Resumen}

La hipoglucemia por hiperinsulinismo endógeno suele presentarse en dos situaciones patológicas: la más frecuente es el insulinoma $y$, en segundo lugar, la nesidioblastosis o síndrome hipoglucémico pancreático no insulinoma. La nesidioblastosis es una causa poco frecuente de hipoglucemia por hiperinsulinismo en adultos. Presentamos el caso de un paciente adulto con cuadros recurrentes de hipoglucemia secundarios a nesidioblastosis.

Palabras clave: Hipoglucemia. Hiperinsulinismo endógeno. Nesidioblastosis. Adulto.

\section{Abstract}

Hypoglycemia due to endogenous hyperinsulinism usually occurs in 2 pathological situations: the most frequent is insulinoma and, secondly, nesidioblastosis or also known as non-insulinoma pancreatic hypoglycemic syndrome. Nesidioblastosis is a rare cause of hyperinsulinic hypoglycemia in adults. We present the clinical case of an adult patient with recurrent hypoglycemia secondary to nesidioblastosis.

Key words: Hypoglycemia. Endogenous hyperinsulinism. Nesidioblastosis. Adult.

\section{Introducción}

La glucosa es el sustrato metabólico clave para el metabolismo celular. En condiciones fisiológicas normales, la concentración de glucosa en plasma se mantiene dentro de un rango estrecho (60-100 mg/dl). La insulina es la hormona principal encargada de disminuir la concentración de glucosa en plasma; en condiciones fisiológicas normales, la secreción de insulina de las células beta se regula con precisión para prevenir la hipoglucemia y la hiperglucemia'.

En la práctica clínica diaria, la causa más común de hipoglucemia es la iatrogénica, causada por insulina o secretagogos de insulina utilizados en el

\section{Correspondencia:}

*Saúl E. Torres-Arano

Calle 117 B diagonal 777 , entre 92 y 94

Los Almendros III

Fecha de recepción: 17-11-2020

C.P. 97314, Ciudad Caucel, Mérida, Yuc., México

E-mail: storres0793@ hotmail.com
Fecha de aceptación: 03-02-2021

DOI: $10.24875 / C I R U .20001253$
Cir Cir. 2021;89(S1):70-75

Contents available at PubMed www.cirugiaycirujanos.com
la licencia CC BY-NC-ND 0009-7411/@ 2021 Academia Mexicana de Cirugía.
(http://creativecommons.org/licenses/by-nc-nd/4.0/). 
tratamiento de la diabetes mellitus ${ }^{2}$. La hipoglucemia se confirma cuando está presente la tríada de Whipple: 1) síntomas o signos compatibles con hipoglucemia; 2) glucosa plasmática $<55 \mathrm{mg} / \mathrm{dl} \mathrm{me-}$ dida con un método preciso, como una muestra de sangre venosa; y 3) resolución de los síntomas al aumentar la glucosa plasmática ${ }^{3}$. La hipoglucemia hiperinsulinémica se presenta como hipoglucemia durante el ayuno, el ejercicio o el periodo posprandial, presentando síntomas principales adrenérgicos (por ejemplo, sudoración, temblor, palpitaciones, taquicardia, agitación o hambre) o neuroglucopénicos (por ejemplo, alteración de la consciencia, del habla o de la memoria, visión borrosa, convulsiones, ataxia o pérdida del conocimiento) ${ }^{1}$. La hipoglucemia por hiperinsulinismo endógeno suele presentarse en dos situaciones patológicas: la más frecuente es el insulinoma y, en segundo lugar, la nesidioblatosis o síndrome hipoglucémico pancreático no insulinoma 4 .

Se presenta el caso de un paciente adulto con cuadros recurrentes de hipoglucemia secundarios a hiperinsulinismo endógeno.

\section{Caso clínico}

Varón de 31 años con antecedente de diabetes mellitus tipo 2 de 1 año de evolución, sin tratamiento, por episodios repetitivos de hipoglucemia; hipertensión arterial de 1 año de evolución tratada con captopril; y depresión tratada con quetiapina y duloxetina. Inició su padecimiento en 2014 con episodios de hipoglucemia repetitivos, requiriendo hospitalizaciones en otro centro, manejado únicamente con solución glucosada. Fue traído a nuestra institución por paramédicos tras presentar un cuadro de 48 horas de evolución de diaforesis, palpitaciones, malestar general y lipotimia sin llegar a síncope, acompañado de hipoglucemia $(55 \mathrm{mg} / \mathrm{dl}$ glucemia capilar), con mejoría tras la administración de soluciones glucosadas (tríada de Whipple). El paciente refirió pérdida de peso de aproximadamente $12 \mathrm{~kg}$ en 2 meses. Por ausencia de sintomatología durante su internamiento, fue egresado. Continuó su seguimiento en la consulta externa de endocrinología, donde se realizó el diagnóstico bioquímico de insulinoma, con valores de insulina de $154 \mu \mathrm{UI} / \mathrm{ml}$ (valor de referencia: 6-27). Fue valorado por el servicio de cirugía general, donde se solicitó una tomografía computada abdominal contrastada que mostró el páncreas aparentemente normal. Se solicitó una
Tabla 1. Valores de insulinemia en relación con el tiempo, posterior a la estimulación intraarterial con $5 \mathrm{ml}$ de gluconato de calcio al $10 \%$

\begin{tabular}{lcccccc}
\hline Arteria & Basal & $\mathbf{3 0} \mathbf{s}$ & $\mathbf{6 0} \mathbf{s}$ & $\mathbf{9 0} \mathbf{s}$ & $\mathbf{1 2 0} \mathbf{s}$ & $\mathbf{1 8 0} \mathbf{s}$ \\
\hline Esplénica & 51.56 & 51.81 & 51.6 & 57.99 & 61.03 & 62.06 \\
Gastroduodenal & 55.49 & 59.94 & 1.04 & 63.14 & 64.89 & 66.69 \\
Hepática propia & 51.24 & 49.35 & 44.97 & 50.51 & 49.73 & 50.22 \\
Mesentérica superior & 49.33 & 49.24 & 48.95 & 49.08 & 39.04 & 49.91 \\
\hline Valores de insulina expresados en $\mu \mathrm{U} / \mathrm{ml}$. & & & &
\end{tabular}

Valores de insulina expresados en $\mu \mathrm{U} / \mathrm{ml}$

resonancia magnética de abdomen que reportó un parénquima pancreático homogéneo, sin tumoraciones. Se realizó una prueba de ayuno prolongado, durante la cual presentó hipoglucemia sintomática con glucemia capilar de $56 \mathrm{mg} / \mathrm{dl}$ en el periodo posprandial y de $49 \mathrm{mg} / \mathrm{dl}$ a la 1:00 a.m.; en ayuno de 5 horas, se tomaron muestras sanguíneas que evidenciaron insulina de $139 \mu \mathrm{Ul} / \mathrm{ml}$ y glucosa de $42 \mathrm{mg} / \mathrm{dl}$, confirmándose el diagnóstico bioquímico de hipoglucemia hiperinsulinémica.

Por sospecha de insulinoma o nesidioblastosis, se realizó cateterismo selectivo de las arterias gastroduodenal, esplénica, hepática propia y mesentérica superior, obteniendo los valores de insulina descritos en la tabla 1.

Durante el seguimiento, el paciente sufrió un traumatismo facial secundario a una caída de la propia altura debida a un síncope secundario a un episodio de hipoglucemia. Por persistir con la sintomatología, se decide intervención quirúrgica bajo la sospecha diagnóstica de nesidioblastosis. Durante la cirugía se realizó pancreatectomía distal laparoscópica preservadora del bazo, sin complicaciones (Figs. 1 a 4). El resultado histopatológico reportó nesidioblastosis del adulto (Figs. 5 a 8). Fue egresado sin complicaciones. Durante los controles en la consulta externa refirió episodios de hiperglucemia manejados con hipoglucemiantes orales (metformina) con adecuado control metabólico, sin nuevos episodios de hipoglucemia documentados hasta el momento.

\section{Discusión}

Como ya hemos mencionamos, la hipoglucemia hiperinsulinémica persistente en adultos generalmente es causada por un insulinoma, pero en raras ocasiones puede estar relacionada con la nesidioblastosis del adulto 5 . 


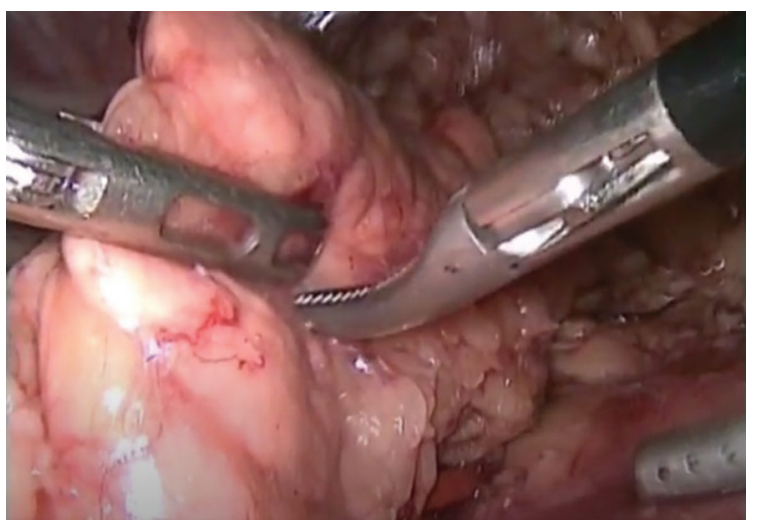

Figura 1. Disección de los medios de fijación del cuerpo y la cola del páncreas.

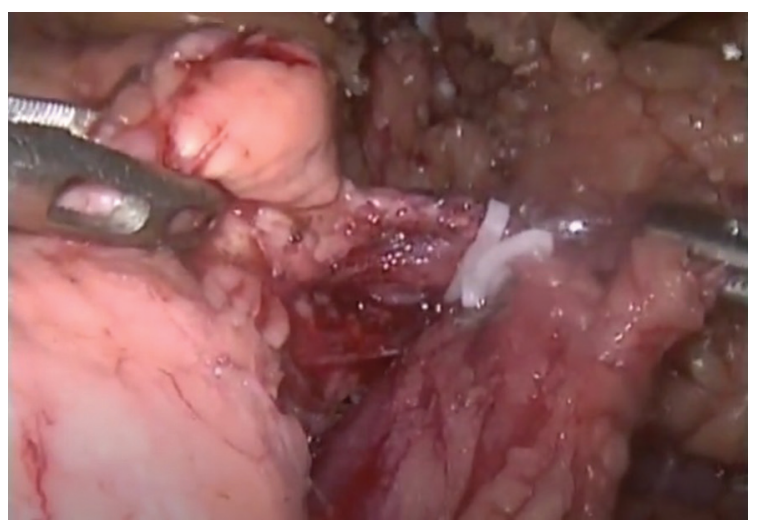

Figura 2. Disección de las arterias nutricias del páncreas y ligadura con Hem-o-lok ${ }^{\circledast}$.

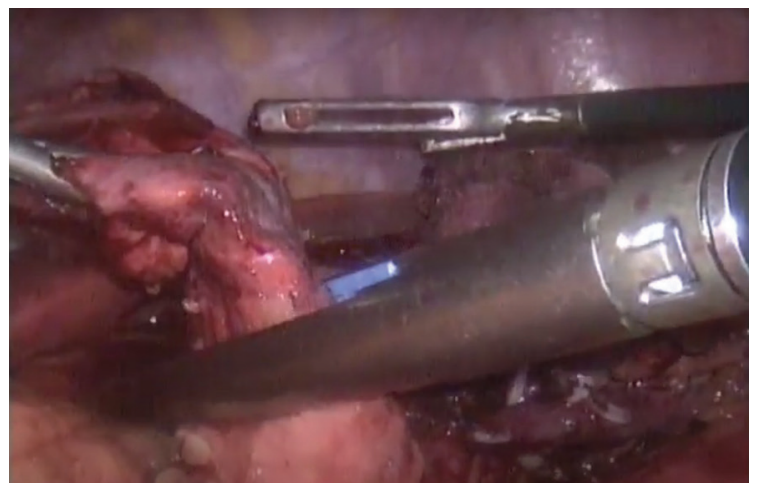

Figura 3. Sección de la cola del páncreas con endoengrapadora lineal.

La nesidioblastosis fue descrita por primera vez en 1938 por George Laidlaw. Este término proviene del griego nesidion, que significa «islas», y explosiones que se refieren a la germinación. Así, Laidlaw la

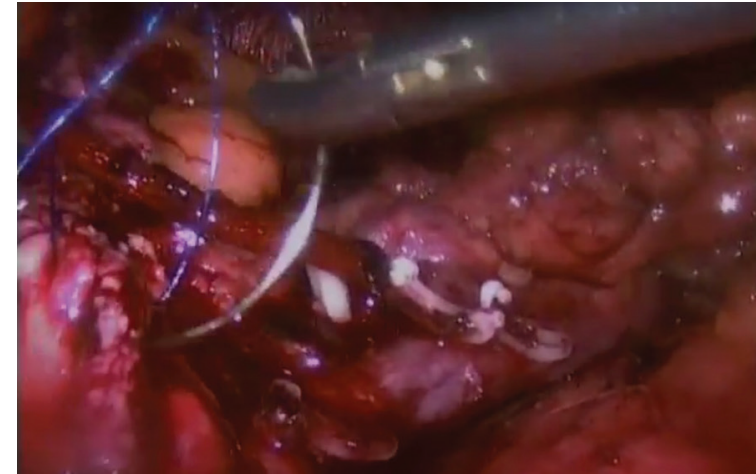

Figura 4. Cierre del muñón pancreático en segundo plano con sutura no absorbible.

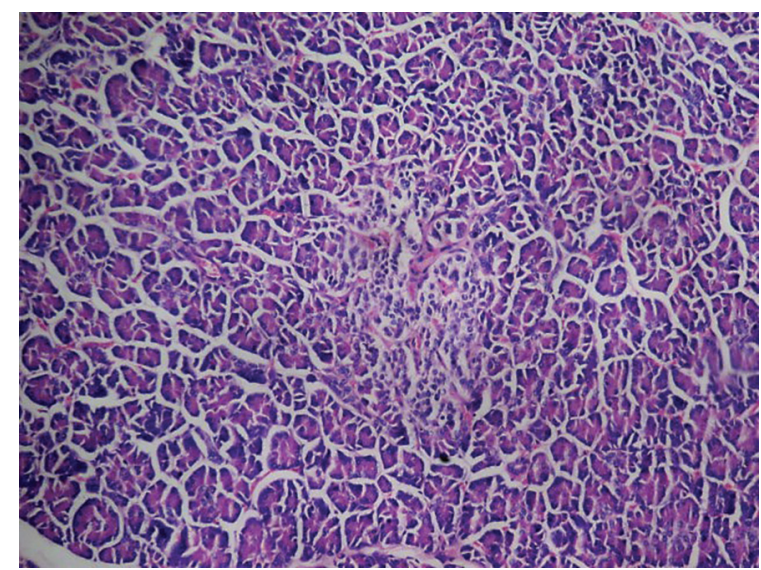

Figura 5. Microfotografía 10x con tinción de hematoxilina-eosina que muestra islotes de Langerhans hiperplásicos en la zona central de la cola del páncreas. Bordes quirúrgicos con islotes sin hiperplasia.

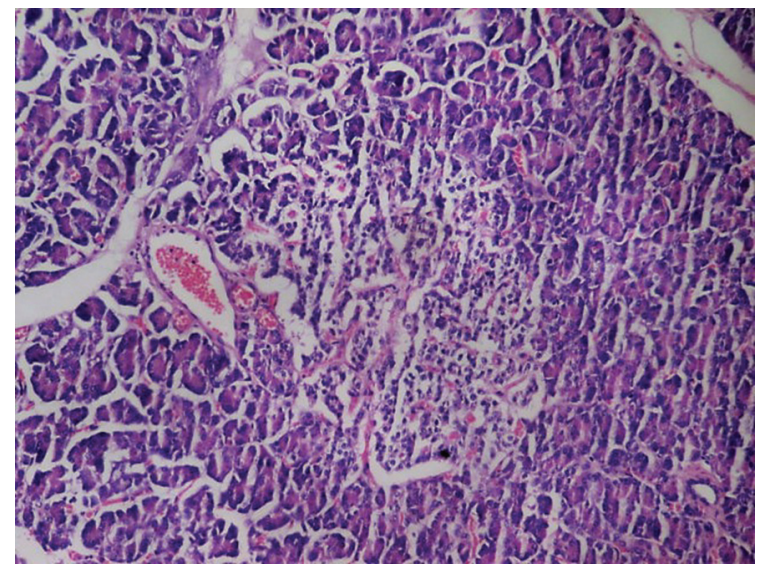

Figura 6. Microfotografía 40x con tinción de hematoxilina-eosina que muestra un complejo de islote y conducto excretor.

definió como un trastorno raro que resulta de la neoformación de células de los islotes de Langerhans en 


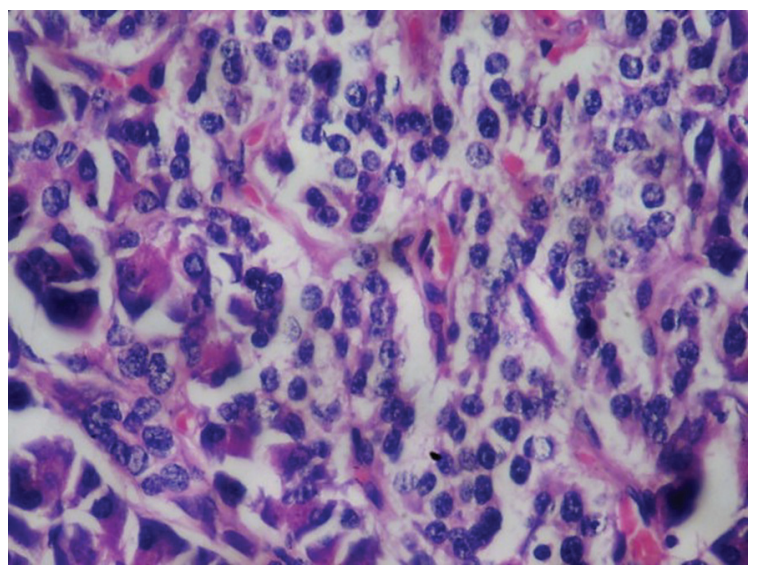

Figura 7. Microfotografía 40x con tinción de hematoxilina-eosina, acercamiento de un islote de aspecto hiperplásico.

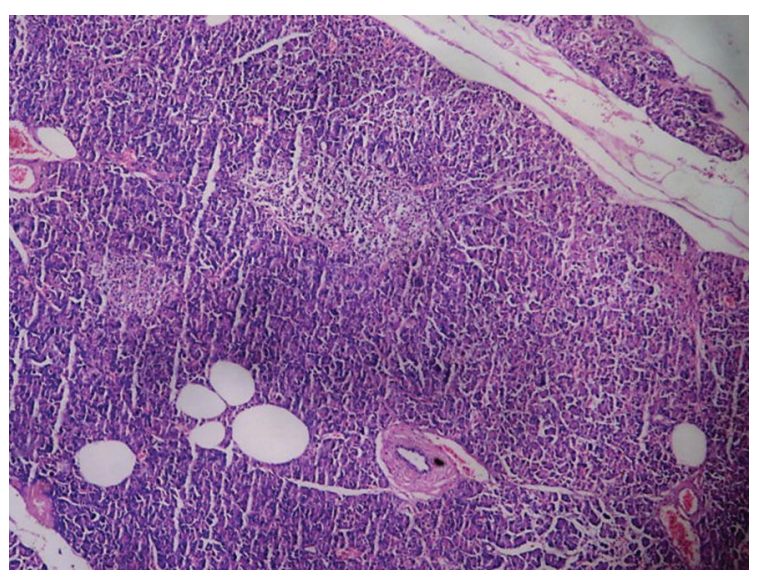

Figura 8. Microfotografía $5 x$ con tinción de hematoxilina-eosina que muestra un complejo de islote y conducto.

el epitelio ductal pancreático exocrino. Inicialmente se observó en recién nacidos, y actualmente es reconocida como la principal causa de hipoglucemia hiperinsulinémica en la niñez ${ }^{6}$.

La nesidioblastosis es una causa poco frecuente de hipoglucemia por hiperinsulinismo en los adultos, representando entre el $0,5 \%$ y el $7 \%$ de los casos $^{5}$. La distinción preoperatoria entre nesidioblastosis e insulinoma puede ser difícil, ya que la presentación bioquímica de ambas es similar y los estudios de imagen son a menudo equívocos ${ }^{6}$. El método por excelencia para realizar el diagnóstico definitivo de hipoglucemia por hiperinsulinismo endógeno es la positividad del test de ayuno a las 72 horas $^{4}$.

La tomografía computada multicorte permite una correlación directa entre el tumor y el resto del parénquima pancreático. Al ser un estudio no invasivo, puede ser considerado de primera línea para la caracterización morfológica de los insulinomas, con una sensibilidad reportada superior al $80 \% 7,8$; sin embargo, en nuestro caso no se observó ninguna alteración en este estudio. Existen otros métodos, como la resonancia magnética y el ultrasonido endoscópico, con sensibilidades y especificidades cercanas al $100 \%$.

Un estudio invasivo disponible para realizar el diagnóstico diferencial entre el insulinoma y la nesidioblastosis es la prueba de estimulación selectiva con gluconato de calcio. Se debe sospechar nesidioblastosis cuando se excluye un insulinoma o una hipoglucemia facticia en todos los procedimientos diagnósticos disponibles, y cuando se demuestre, en una prueba de estimulación selectiva con gluconato de calcio arterial, una respuesta insulínica anormal de todas las partes del páncreas y no solo de una región ${ }^{10}$. Santibañes, et al. ${ }^{4}$ realizaron un estudio descriptivo retrospectivo de pacientes con hipoglucemia por hiperinsulinismo y diagnóstico final de insulinoma o nesidioblastosis, y reportaron que el test de estimulación selectiva con gluconato de calcio tiene una sensibilidad del $87,5 \%$ para localizar o regionalizar un insulinoma de manera preoperatoria. Esta técnica se basa en la inyección intravenosa en bolo de gluconato de calcio en arterias selectivamente cateterizadas: gastroduodenal, esplénica, mesentérica superior y hepática. Posteriormente se recoge una muestra de la vena hepática derecha para medir la insulina a los $0,30,60,90,120$ y 180 segundos posteriores a la infusión. El aumento al doble del valor basal de insulina indica que el tumor está localizado en el área pancreática correspondiente a la arteria evaluada. Por lo tanto, cuando la insulina en plasma aumenta después de la inyección de calcio en la arteria gastroduodenal, es sugestivo de un insulinoma en la cabeza pancreática; cuando el aumento se produce después de la inyección en la arteria mesentérica superior, es sugestivo de un insulinoma en el proceso uncinado; y si el aumento ocurre después de la inyección en la arteria esplénica, es probable que el insulinoma se encuentre en el cuerpo o la cola del páncreas ${ }^{10}$.

A pesar del uso de los métodos diagnósticos mencionados, hasta un $20 \%$ de los insulinomas no se pueden localizar antes de la cirugía, por lo que algunos autores sugieren que no es necesaria la localización preoperatoria, ya que consideran que no hay ninguna técnica superior a la palpación intraoperatoria. 
Es importante considerar que en el $10-20 \%$ de los pacientes falla la localización del tumor en la exploración quirúrgica, si bien esta tasa puede reducirse a menos del $5 \%$ si se asocia el ultrasonido intraoperatorio ${ }^{11-13}$.

La exploración quirúrgica es técnicamente difícil, prolonga la cirugía y aumenta el riesgo de complicaciones, tanto intraoperatorias como posoperatorias ${ }^{14}$. El tratamiento de la nesidioblastosis en los adultos es la resección quirúrgica, pero la línea entre «demasiado» y «muy poco» tejido pancreático es estrecha y no definida ${ }^{15}$. Se aconseja un enfoque prudente e informar al paciente de que pueden ser necesarias varias intervenciones.

No hay un procedimiento óptimo para todos los pacientes adultos con nesidioblastosis, y una segunda operación implica menos riesgo que el de la diabetes mellitus de por vida posterior a una pancreatectomía funcional. Hasta el momento no es posible predecir la recurrencia de la hipoglucemia hiperinsulinémica persistente en los pacientes adultos con nesidioblastosis basándose en las características histopatológicas encontradas en la muestra de resección pancreática ${ }^{10}$.

Tras la resección quirúrgica, la desaparición de los síntomas de hipoglucemia, la normalización de la glucemia y la confirmación histopatológica de la lesión son los criterios utilizados para la curación de estos pacientes ${ }^{11,12}$.

En nuestro caso, el incremento de la concentración de insulina durante el cateterismo selectivo ocurrió en los cuatro vasos canalizados, sugiriendo un proceso difuso, y por lo tanto se decidió el abordaje laparoscópico distal. Si bien se asoció a diabetes posoperatoria, el paciente ya tenía previamente un diagnóstico de diabetes mellitus con episodios alternos.

Gracias a nuestro equipo multidisciplinario fue posible integrar el diagnóstico de nesidioblastosis en el paciente, pero no tenemos registro de otros casos en nuestra unidad. En México no contamos con muchos casos reportados de nesidioblastosis en el adulto. En nuestra búsqueda sobre la incidencia en el país, solo encontramos un caso relacionado con enfermedad renal crónica y un caso aislado concomitante a síndrome de Sheehan. En el caso relacionado con enfermedad renal, el abordaje diagnóstico fue similar al de nuestro caso; en el caso asociado a síndrome de Sheehan, el abordaje diagnostico fue con Octreos$\operatorname{can}^{\circledast}$. Ambos casos convergen en que el tratamiento para la nesidioblastosis es la pancreatectomía distal ${ }^{16,17}$.

\section{Conclusiones}

Bajo la sospecha clínica de nesidioblastosis, el tratamiento médico inicial consiste en una dieta baja en hidratos de carbono en combinación con compuestos como diazóxido, octreotida, acarbosa o bloqueadores de los canales de calcio (como el verapamilo y el nifedipino). Sin embargo, en los casos graves de nesidioblastosis debe ser considerada la pancreatectomía distal, subtotal 0 casi total ${ }^{18}$. Es importante realizar un abordaje diagnóstico preoperatorio completo y descartar todas las causas posibles de hipoglucemia facticia antes de tomar la decisión de intervenir quirúrgicamente a un paciente.

\section{Agradecimientos}

Los autores agradecen profundamente la ayuda y la guía del Dr. Federico López Rosales, ya que sin su orientación este caso no habría sido posible.

\section{Responsabilidades éticas}

Protección de personas y animales. Los autores declaran que para esta investigación no se han realizado experimentos en seres humanos ni en animales.

Confidencialidad de los datos. Los autores declaran que han seguido los protocolos de su centro de trabajo sobre la publicación de datos de pacientes.

Derecho a la privacidad y consentimiento informado. Los autores han obtenido el consentimiento informado de los pacientes y/o sujetos referidos en el artículo. Este documento obra en poder del autor de correspondencia.

\section{Financiamiento}

Los autores declaran que no existe financiamiento.

\section{Conflicto de intereses}

Los autores declaran no tener conflicto de intereses.

\section{Bibliografía}

1. Shah P, Rahman SA, Demirbilek H, Güemes M, Hussain K. Hyperinsulinaemic hypoglycaemia in children and adults. Lancet Diabetes Endocrinol. 2017;5:729-42.

2. Martens P, Tits J. Approach to the patient with spontaneous hypoglycemia. Eur J Intern Med. 2014;25:415-21.

3. Cryer PE, Axelrod L, Grossman AB, Heller SR, Montori VM, Seaquist ER, et al. Evaluation and management of adult hypoglycemic disorders: an Endocrine Society Clinical Practice Guideline. J Clin Endocrinol Metab. 2009;94:709-28. 
4. Santibañes M, Cristiano A, Mazza O, Grossenbacher L, Santibañes E Sánchez R, et al. Síndrome de hipoglucemia por hiperinsulinismo endógeno: tratamiento quirúrgico. Cir Esp. 2014;92:547-52.

5. Witteles RM, Straus IF, Sugg SL, Koka MR, Costa EA, Kaplan EL. Adult-onset nesidioblastosis causing hypoglycemia: an important clinical entity and continuing treatment dilemma. Arch Surg. 2001; 136:656-63.

6. García E, Manzanares M, Padilla D, Villarejo P, Gil A, Muñoz V, et al. Nesidioblastosis. A case of hyperplasia of the islets of Langerhans in the adult. Pancreatology. 2013;13:544-8.

7. Zhao YP, Zhan HX, Zhang TP, Cong L, Dai MH, Liao Q, et al. Surgical management of patients with insulinomas: result of 292 cases in a single institution. J Surg Oncol. 2011;103:169-74.

8. Casanova D, Polavieja MG, Naranjo A, Pardo F, Rotellar F, González F et al. Surgical treatment of persistent hyperinsulinemic hypoglycemia $(\mathrm{PHH})$ (insulinoma and nesidioblastosis). Langenbecks Arch Surg. 2007;392:663-70.

9. Rockall AG, Reznek RH. Imaging of neuroendocrine tumours (CT/MR/ US). Best Pract Res Clin Endocrinol Metab. 2007;21:43-68.

10. Klöppel G, Anlauf M, Raffel A, Perren A, Knoefel WT. Adult diffuse nesidioblastosis: genetically or environmentally induced? Hum Pathol. 2008;39:3-8.
11. Apodaca-Torrez FR, Triviño T, Lobo EJ, Goldenberg A, Figueira A. Insulinomas do pancreas: diagnóstico e tratamento. Arq Gastroenterol. 2003;40:73-9.

12. Tso AW, Lam KS. Insulinoma. Curr Opin Endocrinol Diabetes. 2000;7:83-8.

13. Kasono K, Hyodo T, Suminaga $Y$, Sugiura $Y$, Namai K, Ikoma A, et al. Contrast enhanced endoscopic ultrasonography improves the preoperative localization of insulinomas. Endocr J. 2002;49:517-22.

14. Martínez Hernás S, Pedro T, Sabater L, Argüello L, Real J, Lorente R, et al. Utilidad de la ultrasonografía endoscópica para el diagnóstico de localización de tumores neuroendocrinos pancreáticos: presentación de 2 insulinomas benignos. Endocrinol Nutr. 2007;54:335-9.

15. Raffel A, Krausch M, Anlauf M, Wieben D, Braunstein S, Klöppel G, et al. Diffuse nesidioblastosis as a cause of hyperinsulinemic hypoglycemia in adults: a diagnostic and therapeutic challenge. Surgery. 2007;141:179-84.

16. Ramírez-González LR, Sotelo-Álvarez JA, Rojas-Rubio P, Macías-Amezcua MD, Orozco-Rubio R, Fuentes-Orozco C. Nesidioblastosis en el adulto: reporte de un caso. Cir Cir. 2015;83:324-8.

17. Lozano-Meléndez $E$, Aguilar-Soto $M$, Graniel-Palafox LE, Ceceña-Martínez LE, Valdez-Ortiz R, Solís-Jimenez F. Adult nesidioblastosis in chronic kidney disease. Case Rep Endocrinol. 2019;2019:7640384.

18. Davi MV, Pia A, Guarnotta V, Pizza G, Colao A, Faggiano A. The treatment of hyperinsulinemic hypoglycaemia in adults: an update. J Endocrinol Invest. 2017;40:9-20. 